\title{
EVOLUÇÃO DOS PREÇOS E CUSTOS UNITÁRIOS DA FABRICAÇÃO DO COMPENSADO NO ESTADO DO PARANÁ, BRASIL
}

\author{
EVOLUTION OF PRICES AND UNITARY COSTS OF THE PLYWOOD PRODUCTION IN PARANÁ \\ STATE, BRAZIL
}

Ana Paula Donicht Fernandes ${ }^{1}$ Vitor Afonso Hoeflich ${ }^{2}$ Cláudio José Luchesa ${ }^{3}$ Jorge Antonio de Farias ${ }^{4}$

\section{RESUMO}

Este trabalho analisou a evolução dos componentes do custo da fabricação do compensado e do preço pago aos fabricantes, além de identificar a influência das varáveis macroeconômicas envolvidas. A pesquisa foi realizada com base nos preços e custos do período entre 2005 a 2012. Foram calculados os Índices de Preço com os dados reais médios anuais deflacionados para dezembro de 2012, e para avaliar a relação existente entre os dados e as variáveis macroeconômicas, utilizou-se o coeficiente de correlação de Pearson. Os resultados indicaram o aumento de $0,08 \%$ nos custos reais da cola ureica e a redução de $2,7 \%, 4,7 \%$ 9,9\% e $11,7 \%$ do custo dos insumos: energia elétrica, cola fenólica, serrado e diesel, respectivamente. Todavia, tal diminuição foi bastante inferior se comparada com a diminuição de $26 \%$ do preço recebido pela indústria pelo compensado no período analisado. As lâminas de madeira, no entanto, acompanharam a redução nos preços e tiveram uma queda de $25 \%$, em contrapartida, a mão de obra teve um aumento de $63 \%$. Observou-se uma queda mais acentuada no preço do produto final do que nos custos unitários dos insumos. A análise realizada permite uma compressão da margem de contribuição e a grande influência das variáveis macroeconômicas no preço de venda e nos componentes do custo de produção da fabricação do compensado.

Palavras-chave: economia florestal; índice de preços; margem de contribuição.

\begin{abstract}
This paper analyzed the evolution of the components costs of the plywood production and the prices paid to the industry and also identified the influence of macroeconomic variables in this evolution. The survey was conducted based on costs and prices between 2005 and 2012. The Price Indexes were calculated with the average of the annual real data deflated for December 2012 and, to assess the relation between the data and the macroeconomic variables, Pearson's correlation coefficient was used. The results indicated an increase of $0.08 \%$ in real costs of the ureic glue and a reduction of $2.7 \%, 4.7 \% 9.9 \%$ and $11.7 \%$ of the input costs: electricity, phenolic glue, sawn wood and diesel, respectively. Nevertheless, this decrease in the inputs costs was much lower than the $26 \%$ decrease in the price received by the plywood industry in the analyzed period. The veneer wood, however, followed the reduction in prices and had a fall of $25 \%$; in contrast, the workforce had an increase of $63 \%$. There was a sharper drop in the price of the final product than the unit costs of inputs. The analysis allows a compression of the contribution margin and a large influence of
\end{abstract}

1 Engenheira Florestal, MSc., Doutoranda em Engenharia Florestal pela Universidade Federal do Paraná, Av. Pref. Lothário Meissner, 900, Campus III - Jardim Botânico, CEP 80210-170, Curitiba (PR), Brasil. anapauladfernandes@yahoo.com.br

2 Engenheiro Agrônomo, Dr., Professor da Universidade Federal do Paraná, Av. Pref. Lothário Meissner, 900, Campus III - Jardim Botânico, CEP 80210-170, Curitiba (PR), Brasil. vitor.ufpr@gmail.com

3 Administrador, Dr., Professor do Centro Universitário Curitiba, Rua Chile, 1678, Bairro Rebouças, CEP 80220-181, Curitiba (PR), Brasil. cjluchesa@onda.com.br

4 Engenheiro Florestal, Dr., Professor da Universidade Federal de Santa Maria, Av. Roraima, 1000, Campus Universitário, CEP 97105-900, Santa Maria (RS), Brasil. fariasufsm@gmail.com 
macroeconomic variables in the sale price and in the components of the production cost of manufacturing plywood.

Keywords: forest economics; price indexes; contribution margin.

\section{INTRODUÇÃO}

O compensado é um painel constituído de um conjunto de lâminas coladas com a grã alternada, geralmente em ângulo reto. As lâminas são, usualmente, coladas simetricamente a partir do centro ou miolo do painel, aos pares em ambos os lados (OLIVIERA et al., 2005). Os principais adesivos utilizados na colagem de painéis de madeira são os adesivos sintéticos ureia-formaldeído e fenol-formaldeído (GARCIA et al., 2009).

O compensado é extensamente utilizado na indústria de móveis e construção civil. O seu preço varia conforme a espécie florestal e o adesivo utilizados, a qualidade das faces e o número de lâminas que o compõem. Os compensados apresentam vantagens sobre os demais painéis industrializados, pois são maleáveis e podem ser encurvados (RIBEIRO, 2008). Em 2011, a produção mundial de compensado foi de 83,8 milhões de $\mathrm{m}^{3}$. A China e os Estados Unidos são os maiores fornecedores e respondem por 65,09\% do total mundial, seguidos por Malásia, Indonésia e Rússia; o Brasil é o oitavo maior produtor mundial de compensado (ORGANIZAÇÃO DAS NAÇÕES UNIDAS PARA ALIMENTAÇÃO E AGRICULTURA, 2013).

A base da produção brasileira de compensados é oriunda da Região Amazônica. A madeira de pinus, no entanto, vem ocupando um espaço bastante representativo, a partir de reflorestamentos localizados na Região Sul do País (IWAKIRI et al., 2002).

No Brasil, $36,1 \%$ de toda a madeira produzida são utilizados para a produção de celulose, ao passo que a produção de serrados, a siderurgia a carvão vegetal, os painéis de madeira industrializada e os compensados consomem, respectivamente, $15,2 \%, 10 \%, 7,4 \%$ e $3,7 \%$ do total de madeira. Em relação ao destino do compensado e a distribuição para os mercados internos e externos, em 2012, o volume exportado de compensado foi de $47,1 \%$ e $52,9 \%$ está concentrado no mercado interno, demonstrando, assim, a importância do mercado internacional para o setor florestal brasileiro (ASSOCIAÇÃO BRASILEIRA DE PRODUTORES DE FLORESTAS PLANTADAS, 2012).

O aumento da produção de compensados destinados à exportação torna as empresas suscetíveis às variações na taxa de câmbio e no preço internacional do compensado, sendo esses limitantes para a maior capacidade de investimentos em tecnologia (POLZL, 2010).

Segundo a Associação Brasileira de Produtores de Florestas Plantadas (ASSOCIAÇÃO BRASILEIRA DE PRODUTORES DE FLORESTAS PLANTADAS, 2012), a redução dos mark ups das indústrias de madeira serrada e painéis compensados ocasionada pela redução contínua dos preços reais recebidos pelos fornecedores nacionais e pelo aumento dos custos internos de produção, é a principal razão para perda da competitividade internacional desses segmentos.

Neste contexto, este trabalho avalia em que medida evoluíram os componentes relevantes do custo de fabricação do compensado e o preço de venda deste produto, quando já acabado. A diferença entre a variação do preço de venda e das principais rubricas de custo permitirá avaliar a evolução da margem de contribuição do compensado, elemento essencial na definição das estratégias empresariais. Acessoriamente, o trabalho busca identificar quais as variáveis macroeconômicas que influenciaram tal evolução.

\section{MATERIAL E MÉTODOS}

A evolução dos custos de produção e do preço de venda do compensado foi avaliada com base nos valores históricos entre 2005 a 2012, fornecidos pelo Diretor da empresa STCP Engenharia de Projetos, Bernard François Marie Delespinasse, com sede em Curitiba, Paraná (Os dados são da base de dados da empresa STCP Engenharia de Projetos Ltda $<$ http://www.stcp.com.br/>). As séries históricas compreendem os preços dos fatores unitários dos insumos relacionados na Tabela 1, além do preço de venda das chapas de compensado de pinus $4 \mathrm{~mm}$ e $15 \mathrm{~mm}$ em m3 Ex-Factory (R\$ Médio). 
Os preços históricos, correntes, dos custos unitários e de venda, foram deflacionados para dezembro de 2012, o mês mais recente da série pesquisada. Trabalhou-se, assim, com preços constantes, o que possibilitou analisar a evolução real dos custos unitários dos insumos e do preço de venda. Para o deflacionamento dos preços nominais foi utilizado o Índice de Preços ao Produtor Amplo (IPA), calculado pela Fundação Getúlio Vargas, por ser um índice para análise das variações de preços de produtos agrícolas e industriais.

Assim, com o objetivo de analisar o comportamento das séries históricas destes dados, tanto para a série de preços unitários dos insumos quanto para as séries de preços de venda dos compensados, foram calculados os Índices de Preço com os dados reais médios anuais, com base 100 em dezembro de 2004. Neste caso, um período é escolhido como referência, ou base, e todos os índices são computados em relação aos registros deste período específico. Usualmente, no período base, o índice recebe o valor 100. O númeroíndice é calculado pela razão entre o preço observado de um artigo em um período qualquer e o preço do mesmo artigo no período-base (REIS, 2015 - faltando nas referências). Desse modo foi possível capturar toda a evolução dos preços, desde janeiro de 2005 até dezembro de 2012.

Para este procedimento seguiu-se a recomendação de Viana et al. (2009), que, à medida que transforma valores absolutos (em unidades monetárias) em valores relativos (sem unidade alguma) fixados em uma mesma base, permite que se possa comparar a evolução das diferentes séries históricas de preço.

Este procedimento possibilitou confrontar a evolução do preço de venda do compensado com a evolução dos custos dos seus principais insumos. Desta confrontação resultou a evolução, ainda que parcial, da margem de contribuição da fabricação de compensado. Diz-se parcial porque não se dispunha do coeficiente técnico da produção do compensado. O cálculo da margem de contribuição representa a diferença entre a receita bruta obtida com os produtos e os custos variáveis do sistema de produção (FERNANDES, 2003).

\section{Análise estatística dos dados}

Para avaliar a relação existente entre os dados do trabalho e as variáveis macroeconômicas, utilizou-se o coeficiente de correlação de Pearson, determinando-se com isso, se existe correlação linear significativa entre as variáveis dependentes (preço e custos unitários) e as variáveis independentes (índices macroeconômicos), bem como o grau de associação entre estes pares de variáveis. O teste de significância do coeficiente foi dado pelo teste $t$. A correlação de Pearson foi calculada com a fórmula (SCHNEIDER et al., 2009):

$$
r=\frac{\sum_{i=1}^{n}(X-\bar{X})(Y-\bar{Y})}{\sqrt{\left\{\sum_{i=1}^{n}(X-\bar{X})^{2}\right\}\left\{\sum_{i=1}^{n}(Y-\bar{Y})^{2}\right\}}}
$$

O sinal de $\mathrm{r}$ é dado pela direção da correlação entre as variáveis observadas e calculadas; estará sempre entre -1 e +1 . Esse sinal é obtido pela direção da correlação entre as variáveis (MILONE; ANGELINE, 1995), ou seja:

$\mathrm{r}=+;$ se a correlação for direta, isto é, se o aumento da variável independente implicar um aumento da variável dependente;

$r=-$; se a correlação for inversa, isto é, se o aumento da variável independente implicar uma redução da variável dependente.

Consideram-se agregados macroeconômicos aqueles que dizem respeito ao funcionamento da economia como um todo. Assim, variáveis como Produto Interno Bruto, Taxa de Inflação, Taxa de Desemprego e outros são considerados agregados macroeconômicos pelo fato de não tratarem do comportamento dos agentes econômicos individualmente, centrando sua atenção na soma ou na média dos eventos observados (CAVALCANTI, 1999).

Os parâmetros macroeconômicos selecionados como variáveis relevantes à análise de correlação foram: o PIB, as taxas de juros, câmbio e inflação, o índice do nível de emprego formal e o índice de 
TABELA 1: Componentes associados à fabricação do compensado.

TABLE 1: Components associated with plywood production.

\begin{tabular}{|c|c|}
\hline Insumo & Unidade de Medida \\
\hline Cola fenólica & Kg FOB (R\$ Médio) \\
\hline Cola ureica & Kg FOB (R\$ Médio) \\
\hline Mão de obra - Piso salarial & Do Mês (R\$ Médio) \\
\hline Óleo diesel & Por Litro (R\$ Médio) \\
\hline Energia elétrica industrial trifásica & kw/h (R\$ Médio) \\
\hline Serrado - Pinus - Tábua & $\mathrm{m}^{3}$ Ex-Factory (R \$ Médio) \\
\hline Laminado - Pinus $-2 \mathrm{~mm}$ ou $3 \mathrm{~mm}$ & $\mathrm{~m}^{3}$ Ex-Factory (R\$ Médio) \\
\hline
\end{tabular}

atividade econômica.

A série histórica do PIB Nacional é fornecida pelo Instituto Brasileiro de Geografia e Estatística, esta variável é utilizada quando se objetiva avaliar o nível de atividade econômica de um país. O principal instrumento de política monetária à disposição do Banco Central são as operações de mercado aberto, que lhe permitem controlar as taxas de juros do mercado monetário.

Nesse sentido, optou-se por utilizar a taxa SELIC, como uma proxy da taxa de juros. A taxa de câmbio, o índice de inflação ao consumidor (IPA), o índice do nível de emprego formal e o índice de atividade econômica (IBC-Br) foram obtidos junto à sessão de Indicadores Econômicos do Banco Central do Brasil, considerados indicadores agregados da atividade econômica do país.

Em referência ao relacionamento entre os índices macroeconômicos e os preços e custos unitários da produção do compensado, Bisquerra, et al. (2001) sugerem a seguinte escala de interpretação dos índices de correlação de Pearson (Tabela 2):

\section{RESULTADOS E DISCUSSÃO}

Nos últimos anos, o setor de painéis baseados em florestas plantadas vem experimentando quedas

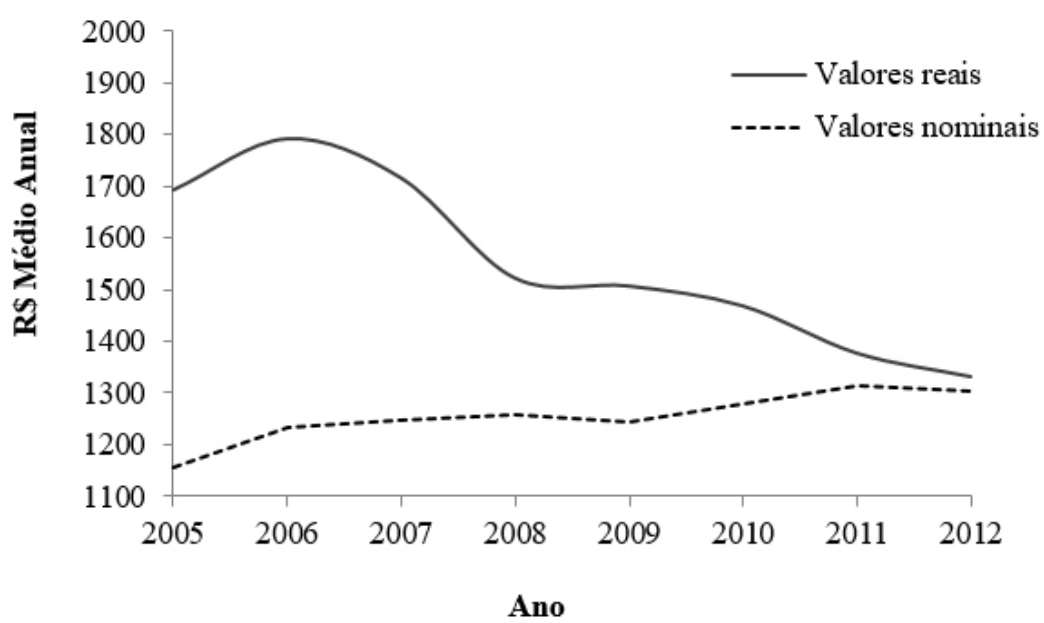

Fonte: Cálculos dos autores a partir de dados primários da STCP Database.

FIGURA 1: Histórico de preços pagos aos fabricantes de compensado entre 2005 e 2012.

FIGURE 1: Historic of the prices paid to plywood producers between 2005 and 2012. 
TABELA 2: Escala de interpretação dos índices de correlação de Pearson.

TABLE 2: Interpretation scale of Pearson's correlation coefficients.

\begin{tabular}{lcc}
\hline & Coeficiente & Interpretação da Correlação $(r)$ \\
\hline$r=1$ & Perfeita \\
$0,80<r<1$ & Muito alta \\
$0,60<r<0,80$ & Alta \\
$0,40<r<0,60$ & Moderada \\
$0,20<r<0,40$ & Baixa \\
$0<r<0,20$ & Muito baixa \\
$r=0$ & Nula \\
\hline
\end{tabular}

constantes nos preços reais pagos aos produtores no Paraná. Em 2006, o preço real pago aos produtores pelo compensado de pinus, para uso geral, com $4 \mathrm{~mm}$, foi de aproximadamente $\mathrm{R} \$ 1.793,00$, apresentando um comportamento declinante até 2012, chegando a R \$ 1.331,96, uma redução de 26\% (Figura 1).

Essa redução constante nos preços reais do compensado, desde meados de 2005, pode ser relacionada à forte competição com os painéis de madeira reconstituída (MDF - Medium Density Fiberboard; e o OSB - Oriented Strand Board), nos setores de móveis e de construção civil, respectivamente. Além disto, a valorização do real frente ao dólar americano e ao euro (início 2007- fim 2012), desacelerou as exportações do compensado para seus principais compradores, Estados Unidos, Reino Unido, Bélgica e Alemanha, aumentando a oferta interna.

A inflação acumulada de janeiro de 2005 a junho de 2012 (período de 78 meses) no Brasil foi de $46,21 \%$, segundo o IPA/FGV. No mesmo período, nominalmente, a cola fenólica e a cola ureica, importantes matérias-primas na fabricação do compensado, variaram, aproximadamente, $40 \%$ e $4 \%$, respectivamente.

O comportamento dos custos unitários dos componentes associados à fabricação do compensado, em preços reais, apresentou reduções inferiores se comparado à redução no preço de venda, como pode ser observado na Figura 2.

Em preços constantes no ano-base, observou-se o aumento dos custos reais de $0,08 \%$ para a cola ureica e a redução de $2,7 \%, 4,7 \%, 9,9 \%$ e $11,7 \%$ dos custos dos insumos: energia elétrica, cola fenólica, serrado e diesel, respectivamente. Uma diminuição bastante inferior se comparada à diminuição no preço recebido pelos fornecedores.

As lâminas de madeira, no entanto, acompanharam a redução nos preços e tiveram uma variação negativa de $25 \%$ no período. Essa diminuição no preço das madeiras laminadas, pode ser associada à crise internacional de 2008 que reduziu os preços, resultante, em parte, pela variação cambial. Em contrapartida, a mão de obra teve um aumento de $63 \%$.

Segundo Pitzahn et al. (2008), o maior problema enfrentado pelas empresas não é a valorização do Real, ou o preço de exportação da madeira serrada e do compensado, mas sim uma elevada inflação setorial de custos, principalmente das matérias-primas (madeira e colas). Porém, esta afirmativa de Pitzahn et al. (2008), não está totalmente correta, conforme os dados apresentados, o que ocorreu, efetivamente, foi uma queda mais acentuada no preço do produto final, do que nos custos dos insumos. Esta evolução comprimiu a margem de contribuição (Tabela 3). 
TABELA 3: Margem de contribuição dos insumos em relação ao preço de venda do compensado, no período entre 2005 e 2012.

TABLE 3: Contribution margin of cost inputs relative to the selling price of plywood, between 2005-2012.

\begin{tabular}{lccc}
\hline Insumo/Produto & Variação Total no período (\%) & Variação média anual (\%) & $\begin{array}{c}\text { Diferença com relação } \\
\text { ao preço de venda }\end{array}$ \\
\hline Preço de Venda & $-26,0$ & $-3,693$ & \\
Laminado & $-25,0$ & $-3,532$ & 0,161 \\
Diesel & $-11,7$ & $-1,469$ & 2,224 \\
Serrado & $-9,9$ & $-1,294$ & 2,399 \\
Cola fenólica & $-4,7$ & $-0,599$ & 3,094 \\
Energia Elétrica & $-2,7$ & $-0,341$ & 3,352 \\
Cola ureica & $-0,08$ & $-0,010$ & 3,683 \\
Mão de Obra & 63,0 & 6,297 & $-2,604$ \\
\hline
\end{tabular}

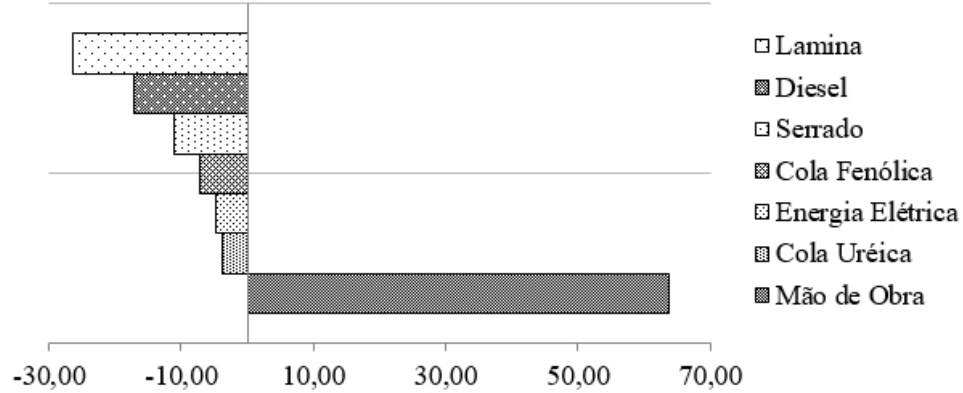

Fonte: Cálculos dos autores a partir de dados primários da STCP Database.

FIGURA 2: Variação no custo dos fatores unitários da fabricação do compensado entre 2005 e 2012.

FIGURE 2: Cost variations of unitary factors on plywood production between 2005 and 2012.

Segundo Associação Brasileira de Produtores de Florestas Plantadas (2013), os principais fatores que influenciam o desempenho na produção do compensado são a política cambial, o ritmo de crescimento das economias nacional e internacional e a crescente concorrência internacional, principalmente a chinesa.

Observando os coeficientes de correlação de Pearson (Tabela 4) verifica-se que o preço de venda do compensado tem relação negativa muito alta com o Produto Interno Bruto, Índice de Inflação (IPA) e o Índice de Emprego $(-0,94,-0,97,-0,94$, respectivamente), indicando que à medida que estas variáveis macroeconômicas aumentam, implicam em uma redução do preço de venda.

Por outro lado, o preço de venda do compensado reage de forma direta à variação da taxa de câmbio $(0,73)$, o que demonstra a grande importância desta variável para a balança comercial brasileira. Movimentos de desvalorizações na taxa de câmbio estão sempre associados com ganhos de saldo comercial, enquanto que períodos de grandes valorizações cambiais acabam por refletir em redução de saldos ou deficit comerciais.

Em relação à interação com os componentes de custos associados à fabricação do compensado, constata-se que as colas, fenólica e ureica, e a mão de obra, se relacionam com os índices macroeconômicos de forma semelhante, relação positiva muito alta, para o PIB, taxa de juros, IPA, índice de emprego e IBC-BR. Inversamente, estes custos se relacionam com a taxa de juros, de forma negativa. Sendo esta variável, uma influenciadora nos custos do capital das empresas e consumidores, estimulando ou desestimulando o consumo e os investimentos na cadeia produtiva.

Os insumos como, as matérias-primas, madeira serrada e laminada, relacionam-se de forma 
TABELA 4: Coeficientes de Correlação de Pearson entre o preço de venda do compensando e os custos unitários dos insumos com as variáveis macroeconômicas.

TABLE 4: Pearson's correlation coefficients between the selling price of the plywood and the costs of inputs with the macroeconomic variables.

\begin{tabular}{lcccrrr}
\hline & PIB Brasil & $\begin{array}{c}\text { Taxa de } \\
\text { Juros }\end{array}$ & $\begin{array}{c}\text { Taxa de } \\
\text { Cambio }\end{array}$ & $\begin{array}{c}\text { Índice de } \\
\text { inflação }\end{array}$ & $\begin{array}{c}\text { Índice de } \\
\text { emprego }\end{array}$ & IBC-Br \\
\hline Preço de venda & $-0,94^{* *}$ & $-0,77^{*}$ & $0,73^{*}$ & $-0,97^{* *}$ & $-0,94^{* *}$ & $-0,91^{* *}$ \\
Cola fenólica & $0,96^{* *}$ & $0,80^{*}$ & $-0,85^{\text {ns }}$ & $0,93^{* *}$ & $0,96^{* *}$ & $0,95^{* *}$ \\
Cola ureica & $0,98^{* *}$ & $0,88^{* *}$ & $-0,78^{*}$ & $0,95^{* *}$ & $0,97^{* *}$ & $0,94^{* *}$ \\
Mão de obra & $0,99^{* *}$ & $0,83^{* *}$ & $-0,84^{* *}$ & $0,98^{* *}$ & $0,99^{* *}$ & $0,98^{* *}$ \\
Diesel & $0,15^{\mathrm{ns}}$ & $0,31^{\mathrm{ns}}$ & $-0,009^{\mathrm{ns}}$ & $0,053^{\mathrm{ns}}$ & $0,11^{\mathrm{ns}}$ & $0,71^{\mathrm{ns}}$ \\
Energia elétrica & $0,86^{* *}$ & $0,97^{* *}$ & $-0,47^{\mathrm{ns}}$ & $0,83^{* *}$ & $0,83^{*}$ & $0,76^{*}$ \\
Serrado & $0,54^{\mathrm{ns}}$ & $0,77^{\mathrm{ns}}$ & $-0,27^{\mathrm{ns}}$ & $0,54^{\mathrm{ns}}$ & $0,51^{\mathrm{ns}}$ & $0,47^{\mathrm{ns}}$ \\
Laminado & $0,43^{\mathrm{ns}}$ & $0,27^{\mathrm{ns}}$ & $-0,64^{\mathrm{ns}}$ & $0,43^{\mathrm{ns}}$ & $0,48^{\mathrm{ns}}$ & $0,55^{\mathrm{ns}}$ \\
\hline
\end{tabular}

Em que $:^{* *}=$ significativo ao nível de $1 \%$ de significância $(\rho$ valor $<0,01) ;{ }^{*}$ significativo ao nível de $5 \%$ de significância $(\rho$ valor $<0,05)$; ns = não significativo.

moderada com o PIB Brasil, IPA, índice de emprego e IBC-Br. De outra forma, o preço da madeira serrada tem baixa relação com a taxa de câmbio, o que pode estar ligado ao crescimento de produção da indústria do processamento mecânico mundial e pela substituição da madeira por outros materiais ou também pelo aumento do consumo no mercado interno, especialmente como infraestrutura na construção civil.

Para a correlação das variáveis macroeconômicas, PIB Brasil, Índice de Inflação, Índice de emprego e IBC-Br, foi significativo, em nível de $1 \%$ de significância, o preço de venda do compensado, a cola fenólica, a cola ureica e mão de obra, sendo que, a correlação foi positiva entre todas as variáveis em questão, exceto o preço de venda da madeira que apresentou correlação negativa.

\section{CONCLUSÕES}

Essa análise dos preços e custos unitários dos insumos, indica que há uma distorção econômica que afeta as operações das indústrias madeireiras.

A redução significativa nos preços pagos aos fornecedores e a redução menos que proporcional dos custos, foram, aparentemente, o principal responsável pela redução da competitividade da indústria de compensado do Paraná no mercado nacional e internacional ao longo dos últimos anos.

A compressão da margem de contribuição ocorre devido à queda mais acentuada no preço do produto final do que nos custos dos insumos.

\section{AGRADECIMENTOS}

Agradecimentos ao diretor da STCP Dr. Bernard François Marie Delespinasse (in memoriam) pela pronta disponibilidade, fornecimento dos dados e relevantes questionamentos, indispensáveis ao desenvolvimento deste trabalho.

\section{REFERÊNCIAS}

ASSOCIAÇÃO BRASILEIRA DE PRODUTORES DE FLORESTAS PLANTADAS. Anuário estatístico da ABRAF 2012 ano base 2011. Brasília: ABRAF, 2012. $150 \mathrm{p}$. ASSOCIAÇÃO BRASILEIRA DE PRODUTORES DE FLORESTAS PLANTADAS. Anuário estatístico da ABRAF 2013 ano base 2012. Brasília: ABRAF, 2013. 148 p. 
BISQUERRA, R.; SARRIERA, J. C.; MARTINEZ, F. Introdução à estatística: enfoque informático com o pacote estatístico. SPSS. Porto Alegre: Artes Médicas, 2001.

CAVALCANTI, R. Cenários Macroeconômicos: uma proposta preliminar para o desenbanco. Salvador: Agência de Fomento do Estado da Bahia, 1999. (Artigo Técnico, 2).

ORGANIZAÇÃO DAS NAÇÕES UNIDAS PARA ALIMENTAÇÃO E AGRICULTURA. Food and Agriculture Organization of the United Nations. 2013. Disponível em:

$<\mathrm{http}$ ://faostat.fao.org/site/626/DesktopDefault.aspx?PageID=626\#ancor $>$ Acesso em: 2 ago. 2013. FERNANDES, A. C. Cálculos na agroindústria da cana-de-açúcar. Piracicaba: STAB, 2003. 240 p.

GARCIA, M. P.; PICCINI, A. A. P.; FERREIRA, E. S. Avaliação da qualidade de quatro resinas comerciais utilizadas na colagem de painéis de madeira reconstituída. In: CONGRESSO DE INICIAÇÃO CIENTÍFICA, 18., 2009, Pelotas. Anais... Pelotas: Imprensa Universitária, 2009.

IWAKIRI, S. et al. Produção de compensados de Pinus taeda L. e Pinus oocarpa Schiede com diferentes formulações de adesivo uréia formaldeído. Revista Árvore, Viçosa, MG, v. 26, n. 3, p. 371-375, 2002.

MILONE, G.; ANGELINI, F. Estatística Aplicada. 1. ed. São Paulo: Atlas, 1995.

PITZAHN, E.; DELESPINASSE, B.; RODRIGUES, R. Entendendo a crise na indústria de serrados e compensados. Informativo STCP, n. 11, 2008.

POLZL, W. B. et al. Perfil produtivo das empresas de madeira compensada no estado do Paraná. Revista Árvore, Viçosa, MG, v. 34, n. 1, p. 189-196, 2010.

RIBEIRO, T. S. Produção de painéis compensados de Pinus taeda com resina uréia-formaldeído utilizando diferentes extensores. 2008. 31 f. Monografia (Curso de Engenharia Florestal) - Instituto de Florestas da Universidade Federal Rural do Rio de Janeiro, Rio de Janeiro, 2008.

SCHNEIDER, P. R.; SCHNEIDER, P. S. P.; SOUZA, C. A. M. Análise de regressão aplicada à Engenharia Florestal. 2. ed. Santa Maria: UFSM, Centro de Pesquisas Florestais, 2009. 294 p.

VIANA, J. G. A.; SOUZA, R. S.; SILVEIRA, V. C. P. Evolução dos preços históricos da bovinocultura de corte do rio grande do sul: tendência e comportamento dos preços em nível de produtor e consumidor. Ciência e Agrotecnologia, Lavras, v. 33, n. 4, p. 1109-1117, jul./ago. 2009. 